\title{
Obesity: a review and commentary
}

\section{Definition and assessment}

The word obesity originates from the Latin term for "overeating." This reflects the age-old bias that etiology is simply gluttony. This ignores the fact that obesity results from an Imbalance between food energy consumption and energy expenditure via physical activity (PA). Further, as discussed later there is strong epidemiological evidence that a sedentary lifestyle plays a central role in the worldwide obesity epidemic In Industrialized societies

The precise assessment of body composition, including fat volume, requires sophisticated and relatively expensive laboratory techniques, such as hydrostatic weighing, dual energy $\mathrm{x}$-ray absorptiometry (DEXA), or bloelectric impedance. Such techniques are impractical for use in large-scale, population-based epidemiological studies. Instead, Indirect anthropometric measurements based on relative body weight for height are generally employed, particularly the body mass index (BMI), expressed in kg of body weight divided by height, squared $\left(\mathrm{kg} / \mathrm{m}^{2}\right.$-In fact, most of the available information about public health implications of obesity are based solely on BMI levels. ${ }^{1}$

The World Health Organization (WHO) and the National Center for Health Statistics uses the BMI to classify selective body weight in adults as follows: ${ }^{2}$
a. Underweight: $<18.5$
b. Normal Weight: 18.5-24
c. Overweight: 25-29
d. Obese Grade 1: 30-34
e. Obese Grade 2: 35-39
f. Obese Grade 3 (severe): 5.40

Relative weight for children and adolescents is also commonly classified by BMI; however, Instead of using fixed values of BMI, classification is based on comparing levels with those corresponding to the relative percentage for healthy young people of the same age and sex. ${ }^{3}$ By this method, children and adolescents (age 2 to 19years) are classified as underweight if their BMI is at the $5^{\text {th }}$ percentile or less for their age and sex; as overweight between the $85^{\text {th }}$ and $95^{\text {th }}$ percentile and obese above the $95^{\text {th }}$ percentile.

A limitation in using BMI as a surrogate measure of both fat volume in adults is that it generally has about a 0.8 correlation with laboratory assessments of percent body fat (\% BF). ${ }^{4-7}$ Thus, its use is associated with a high percentage of both false positives and negatives for obesity. For example, in a study in which men and women were classified as obese by their BMI, only about half of the men and less than two thirds of the women were classified correctly as obese $(\geq 25 \%$ $\mathrm{BF}$ for the men and $\leq 30 \% \mathrm{BF}$ for the women), using bioelectrical impedance assessment. $^{8}$

Further, for men whose BNB was in the overweight category, $20 \%$ had \% BF levels in the lean range $(\leq 20 \%)$ and $10 \%$ had $\mathrm{BF}$ in the obese range $(\geq 30 \%)$. Another easy to asses anthropometric measure associated with body (Indiscernible) is waist circumference (WC). WC of $\geq 35$ Inches $(90 \mathrm{~cm})$ in men and $\geq 40$ Inches $(102 \mathrm{~cm})$
Volume 7 Issue 2 - 2017

\author{
Arthur S Leon \\ School of Kineslology, USA
}

Correspondence: Arthur S Leon, School of Kineslology, Cooke Hail, 1900 University Ave SE Minneapolis, MN 55455, USA,Email leonx002@umn.edu

Received: June 22, 2017 | Published: August 10, 2017

in women are generally associated with excess abdominal visceral obesity. ${ }^{3,9}$ Excess waist circumference and associated visceral fatness are commonly associated with the so-called metabolic syndrome, characterized by dyslipidemia and elevated levels of systolic blood pressure and blood glucose..$^{3,9}$

\section{Epidemiology of obesity}

Based on data from the National Health and Nutrition Examination Study (NHANES), about one of every three of American adults are classified as obese by their BMI and about an equal number are overweight. ${ }^{10}$ Further, one out of every 20 is severely obese (BMI $\geq 40$ ). The prevalence of obesity is the highest among middle aged (40-59years) and older adults ( $>60$ years), as compared to their younger peers (age 20-39years). Ethnic differences in rates also exist in the USA. The highest obesity rates are found among African Americans, followed in descending order by Hispanic, Whites, and those of Asian descent. In addition, there is in North America an Inverse association between socioeconomic status and educational status, and prevalence of overweight and obesity. Further, about one third of American children and adolescents (age 2 to 19years) are classified as overweight or obese. ${ }^{10}$ In a landmark paper Involving a meta-analysis of 230 cohort studies, including 30.3 million participants with 3.74million deaths, overweight and obesity (a BMI $>25$ ) was associated with increased risk of all-cause mortality. The nadir of the distribution curve among nonsmokers was observed to be at BMI 2324.

Obesity rates across all ethnic groups are the highest among middle-age Americans. This is commonly related to an average weight gain of about one pound per year between age 30 and 50 or 55. ${ }^{11}$ This weight gain is generally associated with a progressive increase in waist circumference, reflecting an accumulation of visceral fat. Physiologic adaptation contributing to this "creeping obesity" associated with middle age, include a progressive decline in basal metabolic rate of one to two percent per decade. This is related to a reduction in lean body mass and menopausal hormonal changes in women. Theoretically, this "creeping obesity" would be attenuated or prevented by either a small reduction in food energy intake or a small Increase in daily volume of PA $(<100 \mathrm{kcal} /$ day $)$.

\section{Health implications of obesity}

Pooled data from a number of major prospective cohort studies reveal that overweight and obesity together are responsible for about 300,000 deaths per year among American adults. This makes it the 
second leading cause of preventable death in the USA close behind tobacco use..$^{12}$ The major contributors to excess mortality associated with obesity in all the published Studies are heart disease, strokes, digestive tract diseases (especially gall bladder disease), accidents, and cancer. ${ }^{12}$ Principal cancer sites in obese men are the colon, rectum, and prostate. For women, they are the uterine endometrium, gall bladder, the cervix, the uterus, ovaries, and the breasts.

\section{Contributions of a sedentary lifestyle}

It is beyond the scope of this paper to review all of the etiologic factors contributing to development of obesity. Suffice it to say that genetic, psychosocial, metabolic, and endocrine factors all contribute, in addition to excess food energy intake and low levels of PA. ${ }^{12}$ It is postulated and supported by growing evidence that obesity as a mass phenomenon in affluent, industrialized societies is sociocultural in origin and is primarily related to mass sedentariness. Daily energy expenditure requirements are markedly reduced for the majority of people in affluent societies, as the result of extensive mechanization and automation, motorized transportation, and labor and setup-saving devices in the home and at work. Furthermore, most of leisure time is commonly spent in passive recreational pursuits, particularly increase screen time In front of a computer or TV. In addition, cross-sectional comparison of dietary energy intake of obese as compared to matched non-obese individuals, have generally failed to find significant differences in dietary energy intake. ${ }^{11}$ Further, the US Department of Agriculture's food survey data reveals a progressive decline in available food for consumption since 1909, while the prevalence of obesity was steadily increasing. ${ }^{11}$

Based on these data, it is concluded that a sedentary lifestyle is the major contributor to the obesity epidemic in this and other affluent countries. Ultimately, however, overweight and obesity results from a prolonged positive energy balance with about one pound of fat stored for about each $3500 \mathrm{kcal}$ of excess energy intake. As previously mentioned, the average American adult gains about one pound a year between age 30 and 50 or 55 . This reflects a positive energy balance of much less than $100 \mathrm{k} \mathrm{cal}$ per day. Also as mentioned, there is strong, consistent observational evidence that paucity in daily PA is the principal contributing factor. Further, USDA annual surveys of public food consumption has shown a steady decline since 1909, while the prevalence of obesity has progressively increased. ${ }^{11}$ This provides strong evidence that reduced energy expenditure rather than overeating is the major contributor to overweight and obesity in the USA.

Moreover, large scale longitudinal observational studies have reported an inverse association of PA and BMI. ${ }^{11}$ In addition, crosssectional studies have generally failed to confirm any significant difference in food energy intake between obese and their matched non-obese peers. ${ }^{11}$

American College of Sports Medicine (ACSM) guidelines recommend a more reasonable PA volume for obesity prevention, management, and weight loss maintenance (ie 30-60minutes daily for prevention and management of obesity and 80-90minutes of moderate intensity PA daily for maintenance of lost weight. Ideally, this should be combined with a modest reduction in dietary energy intake. ${ }^{16}$ Multiple physiological mechanisms are postulated to contribute to both loss of excess weight and healthy promotion of regular PA. These include, in addition to the calories burned during the PA, post-exercise thermogenesis (a transient Increase in resting energy expenditure). Ideally this should be combined with a modest reduction in dietary energy intake. Resistance training several days per week is also recommended to help preserve lean body mass during weight reduction. ${ }^{13}$

The NIH recommended overall goal for management of obesity is to attain a gradual weight reduction of $5 \%$ to $10 \%$ of baseline weight over a 6 month period. If Intensive Intervention fails or in presence of severe obesity or significant co-morbidity, the addition of pharmacologic treatment or bariatric surgical procedures are options.

\section{Obesity management}

The management of obesity requires long-term lifestyle and behavioral changes, and in addition, include medications and/ or bariatric surgical procedures for selected patients. Currently recommended lifestyle changes include a modest reduction in dietary energy (i.e., $500-750 \mathrm{kcal}$ ) with a goal of $5 \%$ to $10 \%$ loss of weight in 6months, on increase in regular aerobic PA, and behavioral modification. Although specific dietary restriction programs alone are generally successful in producing a significant weight loss over a short term (6-12months), only a relatively small number of dieters maintain the lost weight for longer periods of time (i.e. $2 \%$ to $20 \%$ ). Lifestyle behavioral modifications, including eating and PA habits are essential for prevention of obesity, and loss and maintenance of excessive body weight. Behavioral modifications Include helping the overweight or obese person understand the principals of weight regulation, obesity and its detrimental health consequences, and directly address behaviors that require change to promote successful weight maintenance or loss of excess weight. These Include the following: ${ }^{14,15}$

a. Self-monitoring of daily energy Intake and expenditure by PA

b. Rational goal setting (e.g. $5 \%$ to $10 \%$ reduction in weight over 6-12months)

c. Reducing purchase and consumption of tempting high energydense foods

\section{d. Decreasing food portion sizes}

e. Avoiding snacks between meals and at bedtime or limiting them to low calorie foods (eg. celery sticks or carrots)

f. Non-food rewards for successful weight reduction or maintenance

\section{g. Lifestyle relapse prevention}

The modern lifestyle is a major contributing factor to the obesity epidemic. Prevention and management of obesity, and maintenance of weight loss, requires permanent lifestyle behavioral changes. These include, in addition to changes in eating habits, Incorporation of regular PA into the daily routine. This requires reducing time spent sitting, increasing activities of daily living, and performing at least 150-300minutes per week of walking or other moderate Intensity aerobic activities. It should be noted that regular aerobic PA, even in the absence of weight reduction, has multiple favorable health benefits. These beneficial effects are summarized in Table 1 .

It has been demonstrated in a limited number of controlled studies (Including in my laboratory) that a large volume (e.g., 60 to 90minutes, five times per week) of supervised aerobic exercise (eg, treadmill walking or jogging) at an energy expenditure of 2000 to $3500 \mathrm{kcal} /$ week general results in a sustained weight loss. 
Table I Possible beneficial effects of regular dynamic endurance-type PA

\section{Cardiovascular-respiratory adaptations}

Reduced heart rate and blood pressure

Increased aerobic capacity (V02 mite

increased myocardial vascularity and coronary artery size

Increased resistance to ventricular fibrillation

Decreased tendency to thrombosis

Reduced work of breathing

\section{Metabolic adoptions}

Improved blood lipid Ilpo-protein profile

Improved glucose-Insulin dynamic

Increased use of fat for fuel

Reduced all-cause and (?) mortality

Psychosocial benefits

Change In detrimental health habits

Feeling of well-being

improved self-control and self-confidence

Tranquilizing and muscle relaxant effect

Improved stress tolerance

Reduced mental depression

\section{Pharmacologic therapies}

Several types of medications are currently approved by the FDA for use along with comprehensive lifestyle management of obesity. ${ }^{13}$ These include the following:

a. Noradrenergic appetite suppressants, including diethylpropion (Tenuate), phentermine (Fastin), and phenylpropanolamine (Dexatrin)

b. Serotonergic appetite suppressants, including fenfluramine (Pondimin) and dexfenfluramine (Redux)

c. Sibutrannine (IVIeridia), an agent which has both an anorectic effect by inhibiting reuptake of both serotonin and catecholamines, and possible by stimulating thermogenin.

d. Orlistat (Xenical), a pancreatic inhibitor that acts on the gastrointestinal tract to decrease fat absorption. Guidelines for the use of these pharmacologic agents are available..$^{13}$

These different types of drugs are effective in increasing the percentage of obese patients achieving a goal of a $5 \%$ to $10 \%$ loss of their baseline weight over a six -month period. However, they all have many adverse side effects and maintenance of lost weight remains a problem when they are discontinued. In fact, Orlistat is the only one of them currently approved by the FDA for long term use.

\section{Bariatric surgical intervention}

When Intensive lifestyle and medical interventions fall and in persons with severe obesity or serious coexisting medical conditions, surgical intervention is often prescribed. ${ }^{13-16}$ Vertical banded gastro plasty to reduce stomach volume and Roux-en-Y gastric bypass are associated with long term weight loss and reduces all cause mortality as compared to standard weight reduction methods. However, there is a small (about 1\%) surgical mortality rate and persistent gastrointestinal side effects following these procedures.

\section{Summary and conclusions}

Thu following are key points emphasized in this review pertaining to obesity:

a. It is a highly prevalent public health problem in the USA and other western countries; and

b. Associated major health concerns include dyslipidemia, typo 2 diabetes, hypertension, coronary heart disease, strokes, certain types of malignancies, as well as all cause premature mortality.

c. Observational epidemiologic studies clearly also indicate that a sedentary lifestyle is generally an important contributor to the etiology of this condition.

d. Currently, public health experts recommend 150minutes or more per week of moderate intensity physical activity or 15.minutes par week of more vigorous PA as a preventative measure with a goal of a $5 \%$ to $10 \%$ weight reduction over a 6 to 12 month period. Further, 60 to 90 minutes or more daily PA are recommended for maintenance of lost weight.

\section{Acknowledgements}

None.

\section{Conflict of interest}

The author declares no conflict of interest.

\section{References}

1. National Heart, Lung, and Blood institute. Clinical guidelines on the identification, evaluation, and treatment of overweight and obesity in adults. The Evidence Report, NIH Publications No. 98-4083; 1998.

2. Flegal KM, Kit BK, Organs H. Association of all-cause mortality with overweight and obesity using standard BMI categories, a systematic review and meta-analysis. JAMA. 2013;309(1):71-82.

3. Ogden OL, Carroll MN, Regal KM. Prevalence of obesity and trends in BMI among US children and adolescents, 1999-2010. JAMA. 2012;307(5):483-490.

4. Cler MAC, Depres JP, Davies N, et al. Assessing adiposity: A scientific statement from the American Heart Association. Circulation. 2014;124(18):1996-2014.

5. Ranasingbe C, Gamage P, Katulanda P, et al. Relationships between BMI and body fat percentage estimated by bioelectrical impedance in a group of Sri Lankan adults. A cross-sectional study. EMIG Public Health. 2011:13:709-730.

6. Meevunse S, Horgan GW, Elia M. The relationship between BMI and percent body fat, measured by bioelectrical impedance in a large adult sample and referenced by sex and age. Clinical Nutr. 2010;29(5):560560 .

7. Rothman KJ. BMI-related error in the measurement of obesity is curvilinear. Intern J Obesity. 2008;32:556-559.

8. Denrenberg P, Andreoll A, Kukkonen-Harjua K, et al. The validity of predicted body fat percentage from BMI and from impedance in samples of five European populations. Euro Cl Nutr. 2001;55(11):973-979. 
9. Cormier MA, Depres JP, Davis N, et al. Assessing adiposity. A scientific statement from the American Heart Association. Circulation. 2011;124(18):1996-2019.

10. Ogden CL, Carroll MD, Fryar CD, Flegal KM Prevalence of obesity among adults and youth, United States, 2011-2014. CHS Data Brief. 2015;219:1-8

11. Leon AS. The role of physical activity in the prevention and management of obesity. In: Ryan Al, Allman, editors. Sports Medicine. 2nd ed. USA Academic Press Inc; 2002. p. 593-617.

12. Anne D, Sen A, Prasad M. BMI and all cause mortality: Systematic review and non-kinear dos-response meta-analysis of 230 cohort studies with 3.74 million deaths among 30.3 million participants. BMI. 2016;353:1256-1295.
13. Apoyan CM, Garvey WI, Ryan OH. Challenging obesity patients, providers, and expert perspective on the roles of available and emerging nonsurgical techniques. Obesity. 2015;23(Supple S2):1S-26S.

14. Yumuk V, Tsigos C, Fried M, et al. European guidelines for obesity management in adults. Obesity Facts. 2015;8(6):402-424.

15. Garvey WT, Garber Al, Mechanick JI. Medical society guidelines for treatment of obesity. American Association of Clinical Endocrinologists/ American College of Endocrinology. Endorine Practice. 2016;22(Supple 3):1-203.

16. Donsill J, Blair S, Jakicla MA. Appropriate PA intervention strategies for weight loos and prevention of weight regain in adults. Mod Sci Sports. 2009;41:459-471. 